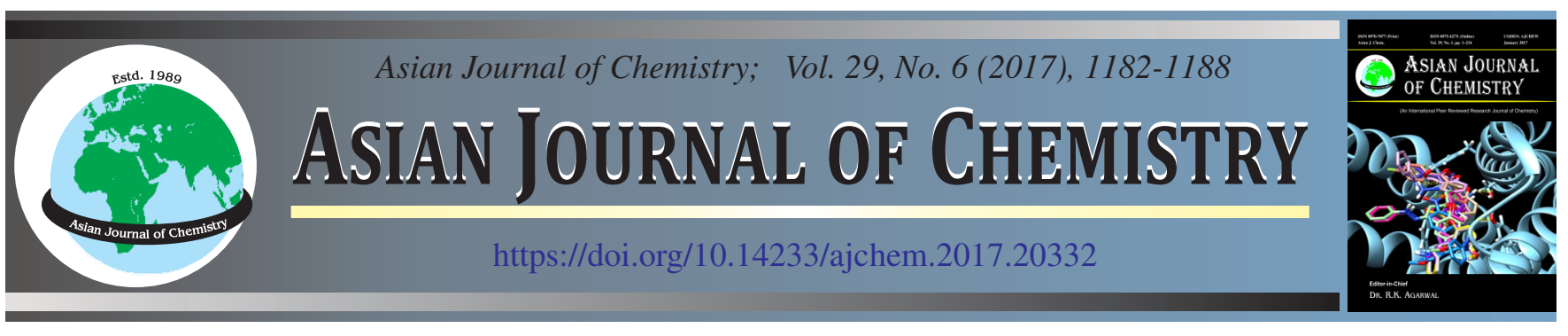

\title{
Growth, Linear and Non-Linear, Dielectrics, Microhardness, Refractive Index and Laser Damage Threshold Studies on Urea Phosphoric Acid Single Crystal
}

\author{
N. Hema, R. Usha, D. Shalini, V. Revathi Ambika and D. Jayalakshmi*
}

Department of Physics, Queen Mary's College (A), Chennai-600 004, India

*Corresponding author: E-mail: hemavenkatesaprabu@yahoo.co.in

\begin{abstract}
An organic new non-linear optical urea phosphoric acid single crystal has been grown by slow evaporation technique. The cell parameters of urea phosphoric acid single crystal and crystalline perfection were confirmed by single and high resolution X-ray diffraction analyses. The presence of functional groups was identified by FT-IR spectral analysis. The UV-visible spectral studies showed that the urea phosphoric acid crystal has wide transmission in the entire visible region. The dielectric property of urea phosphoric acid crystal revealed the normal dielectric behaviour. Mechanical strength of urea phosphoric acid crystal was studied. The refractive index of urea phosphoric acid crystal was also measured. The laser damage threshold value of urea phosphoric acid crystal was found by Nd:YAG laser. The photoconductivity studies were taken. The second order non-linear coefficient of the grown crystal was also measured for urea phosphoric acid single crystal.
\end{abstract}

Keywords: Synthesis, Solubility, X-ray diffraction, FTIR, UV-visible spectra, Second harmonic generation.

\section{INTRODUCTION}

In the recent era, an organic non-linear optical (NLO) crystal is attracting a great deal of attention because of their high non-linearity. These crystals also give rapid response in electro optic modulation, SHG, frequency mixing and optical para-metric oscillation than the inorganic non-linear optical material [1-3]. These are polar crystals and the macroscopic properties reflect the internal asymmetric relationship. The structure of organic chromophores easily modifiable through chemical synthesis to increase the molecular hyperpolarizability, compared to the difficulties of the engineering route of inorganic materials, in which the requirements of noncentrosymmetry and high susceptibility have to be accounted [4,5]. In the present investigation, growth, thermal, optical, laser damage threshold dielectric and microhardness have been carried out on the urea phosphoric acid single crystal.

\section{EXPERIMENTAL}

Characterizations: The grown urea phosphoric acid crystals were subjected to X-ray diffraction studies using Bruker kappa APEXII single crystal X-ray difffractometer, using $\operatorname{MoK}_{\alpha}(\lambda=0.71073 \AA)$. The various functional groups of urea phosphoric acid crystal were identified by the $\mathrm{KBr}$ pellet technique using a Perkin Elmer FTIR spectrometer in the range $4000-450 \mathrm{~cm}^{-1}$. The transmission spectrum of the urea phosphoric acid crystal was studied in the range 200$900 \mathrm{~nm}$ by Perkin Elmer spectrometer. The dielectric measurement has been carried out using HIOKI 3532-50 LCR HITESTER instrument. Microhardness measurement of urea phosphoric acid crystal was carried out using Leitz-Wetzlar Vickers' microhardness tester fitted with a diamond pyramidal indenter attached to an optical microscope. The laser damage threshold value of urea phosphoric acid was measured using Nd:YAG laser with $8 \mathrm{~ns}$ pulse width and $10 \mathrm{~Hz}$ repetition rate. The Kurtz and Perry powder technique was employed on urea phosphoric acid crystal to measure the second harmonic generation efficiency using Nd:YAG laser.

Synthesis: Urea phosphoric acid was synthesized using AR grade urea and phosphoric acid in a stoichiometric ratio of $1: 1$. The reactants were dissolved in double distilled water and was thoroughly mixed using a magnetic stirrer at a constant temperature of $45^{\circ} \mathrm{C}$. A white crystalline salt was obtained. The synthesized salt was further purified by repeated crystallization. The reaction scheme is illustrated as:

$$
\mathrm{CO}\left(\mathrm{NH}_{2}\right)_{2}+\mathrm{H}_{3} \mathrm{PO}_{4} \longrightarrow \mathrm{CO}\left(\mathrm{NH}_{2}\right)_{2}+\mathrm{H}_{3} \mathrm{PO}_{4}
$$

Solubility: The solubility for urea phosphoric acid has been determined for the following four temperatures, 35, 40, 45 and $50{ }^{\circ} \mathrm{C}$. The process was determined by dissolving the solute in water in an air tight container maintained at a constant temperature with continous stirring. After attaining the satu- 
ration, the equilibrium concentration of the solute was analyzed gravimetrically. Same process was repeated and the solubility curve for different temperatures were drawn. Urea phosphoric acid exhibits fair solubility and a positive solubility temperature gradient in water (Fig. 1).

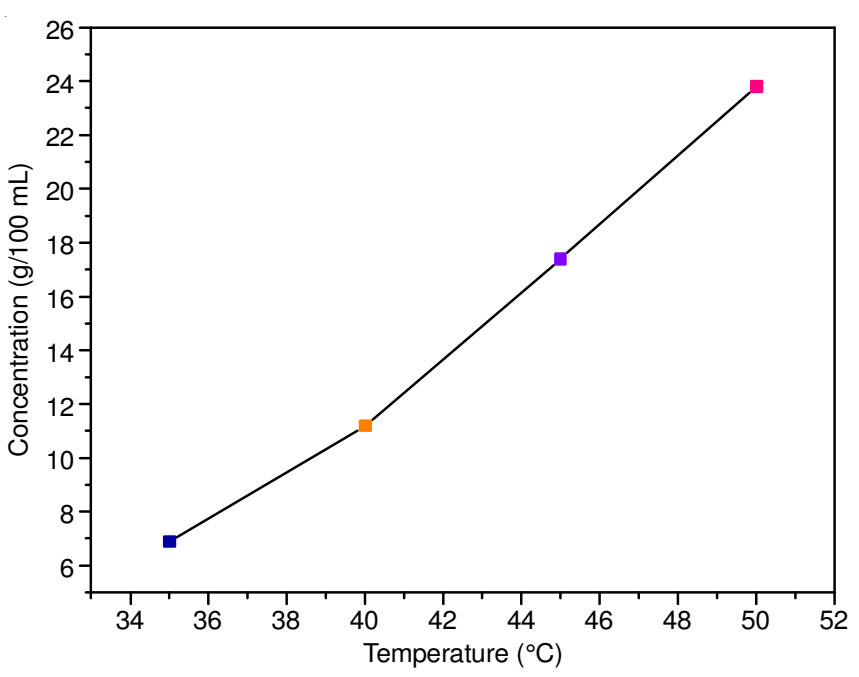

Fig. 1. Solubility curve of urea phosphoric acid

Crystal growth: The saturated solution was prepared in accordance with the solubility curve. Single crystals of urea phosphoric acid were grown from their aqueous solution using the slow evaporation method. The prepared solution was kept in a constant temperature water bath of accuracy of $\pm 0.01^{\circ} \mathrm{C}$. After a span of 19 days, a medium size single crystal with the dimensions of $14 \mathrm{~mm} \times 5 \mathrm{~mm} \times 4 \mathrm{~mm}$ was harvested. The photograph of the grown crystal of urea phosphoric acid is shown in Fig. 2.

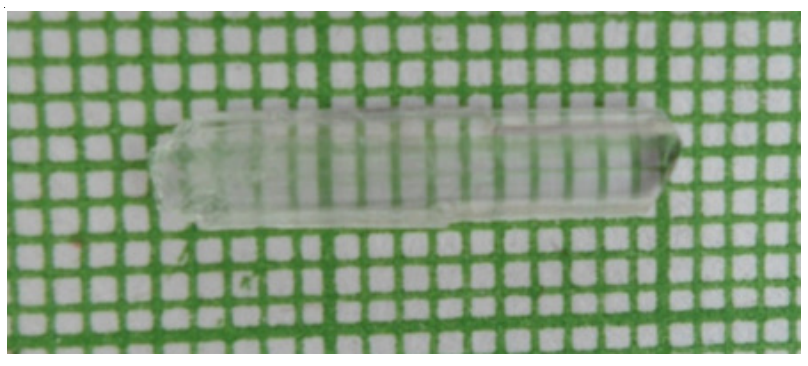

Fig. 2. Photograph of urea phosphoric acid crystal

\section{RESULTS AND DISCUSSION}

Single X-ray diffraction analysis: The cell parameters of the urea phosphoric acid crystal were estimated by single crystal X-ray diffraction analysis and cell parameters are $\mathrm{a}=$ $17.3813 \AA, b=7.425 \AA, c=8.9323 \AA, V=1158 \AA^{3}$, with angle $\beta=\alpha=\gamma=90^{\circ}$ [6]. The grown crystal belongs to orthorhombic crystal system with space group Pbca. It is observed that lattice parameter values and cell volume of urea phosphoric acid are in good agreement with the reported values [7].

High resolution $X$-ray diffraction analysis: The crystalline perfection of the grown urea phosphoric acid single crystal was characterized by HRXRD by employing a multicrystal X-ray diffractometer. The well-collimated and mono- chromatic $\mathrm{MoK}_{\alpha 1}$ beam obtained from the three monochromator Si crystals set in dispersive configuration has been used as the exploring X-ray beam. The specimen can be rotated about the vertical axis, which is perpendicular to the plane of diffraction, with minimum angular interval of $0.4^{\circ}$ arc sec. The diffraction curves were recorded by changing the glancing angle (angle between the incident X-ray beam and the surface of the specimen) around the Bragg diffraction peak position $\theta_{\mathrm{B}}$ starting from a suitable arbitrary glancing angle and ending at a glancing angle after the peak so that all the meaningful scattered intensities on both sides of the peak are included in the diffraction curve. The diffractive curve (DC) was recorded by $\omega$ scan where in the detector was kept at the same angular position $2 \theta_{\mathrm{B}}$ with wide opening for its slit. Before recording the diffraction curve to remove the non-crystallized solute atoms which remained on the surface of the crystal and the possible layers which may sometimes form on the surfaces on crystals grown by solution methods and also to ensure the surface planarity, the specimen was first lapped and chemically etched.

Fig. 3 shows the diffractive curve recorded for a urea phosphoric acid crystal grown by slow evaporation method using $\left(\begin{array}{lll}0 & -1 & 0\end{array}\right)$ diffracting planes under identical experimental conditions. As seen in Fig. 4 the diffractive curve is quite sharp without any satellite peaks showing that the crystal is free from structural grain boundaries. Thus the curve is extremely sharp having full width at half maximum (FWHM) of $30^{\circ}$ arc sec as expected for a nearly perfect crystal from the plane wave dynamical theory of X-ray diffraction. The single sharp diffraction curve with very low FWHM and the absence of scattered intensity (revealing very low density of point defects and their aggregates) along both the wings of the diffractive curve indicates that the crystalline perfection is extremely good. The specimen is a nearly perfect single crystal without having any internal structural grain boundaries.

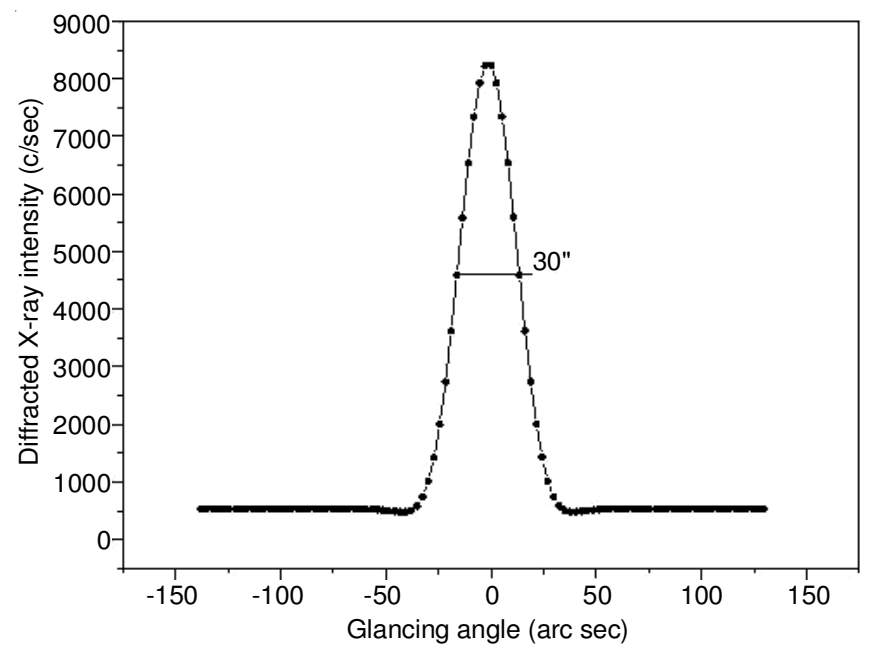

Fig. 3. Diffraction curve for urea phosphoric acid crystal

FTIR studies: The FTIR analysis of urea phosphoric acid was carried out between 4000 and $450 \mathrm{~cm}^{-1}$ using Perkin Elmer model spectrometer. The FTIR spectrum is shown in Fig. 4. The peak at $3778 \mathrm{~cm}^{-1}$ may be assigned to $\mathrm{NH}$ asymmetric stretching. The peaks at 3450 and $1662 \mathrm{~cm}^{-1}$ are assigned to 


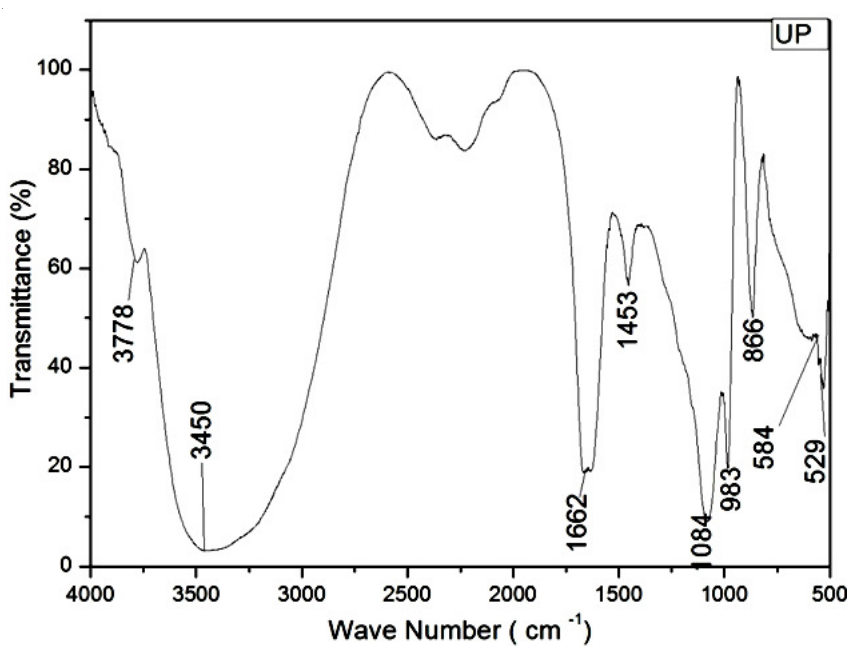

Fig. 4. FTIR spectrum of urea phosphoric acid crystal

$\mathrm{OH}$ asymmetric stretching and $\mathrm{C}=\mathrm{O}$ asymmetric stretching respectively. The peak at $1453 \mathrm{~cm}^{-1}$ is assigned to $\mathrm{P}=\mathrm{O}$ bending. The C-N asymmetric stretching appears at $1084 \mathrm{~cm}^{-1}$. The peaks at $983,866,584$ and $529 \mathrm{~cm}^{-1}$ were attributed to $\mathrm{NCN}$ symmetric stretching, $\mathrm{P}-\mathrm{O}$ asymmetric stretching, $\mathrm{P}=\mathrm{O}$ bending and $\mathrm{P}-\mathrm{O}$ bending vibration respectively. The $\mathrm{C}-\mathrm{N}$ rocking exhibit at $508 \mathrm{~cm}^{-1}$.

UV-visible spectral studies: UV-visible transmission spectrum of urea phosphoric acid crystal was recorded in the range of 190-900 nm using $0.93 \mathrm{~mm}$ thickness crystal sample. Urea phosphoric acid crystal is active in the UV-visible region. It has good transparency of about $55 \%$ with lower cut-off wavelength $278 \mathrm{~nm}$ (Fig. 5a).

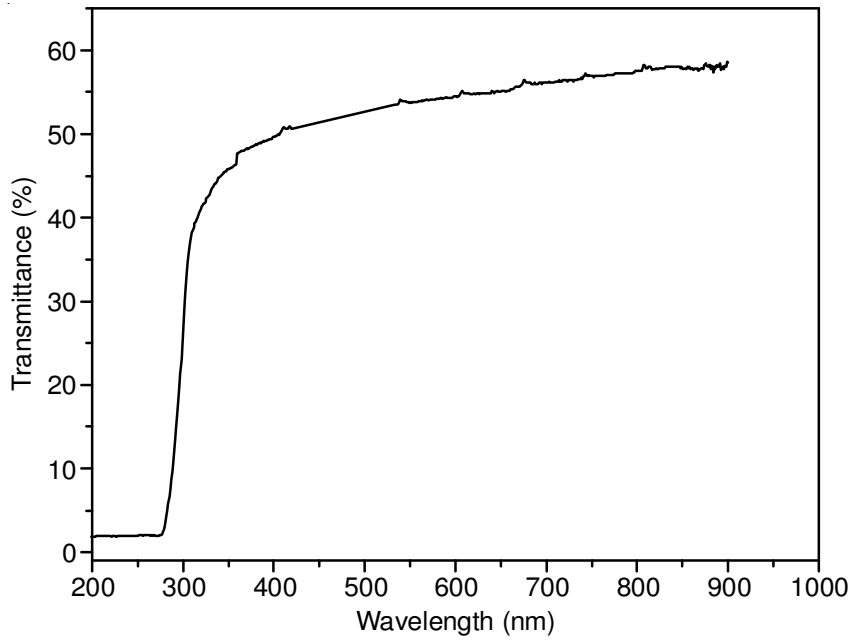

Fig. 5(a). UV-visible transmission spectrum of urea phosphoric acid crystal

The absorption coefficient $(\alpha)$ and the optical parameters such as refractive index $(\mathrm{n})$, reflectance $(\mathrm{R})$ and extinction coefficient $(\mathrm{K})$ have been determined from the transmission (T) spectrum based on the following relations:

$$
\alpha=\frac{2.3026}{\mathrm{t}} \log (1 / \mathrm{T})
$$

where $\mathrm{T}$ is the transmittance and ' $\mathrm{t}$ ' is the thickness of the crystal.

The absorption coefficient ' $\alpha$ ' is related to the extinction coefficient $\mathrm{K}$ by:

$$
\mathrm{K}=\frac{\alpha \lambda}{4 \pi}
$$

The reflectance $(\mathrm{R})$ in terms of the absorption coefficient and refractive index (n) can be derived from the relations:

$$
\begin{gathered}
\mathrm{R}=\frac{\exp (-\alpha \mathrm{t}) \pm \sqrt{\exp (-\alpha \mathrm{t}) \mathrm{T}-\exp (-3 \alpha \mathrm{t}) \mathrm{T}+\exp (-2 \alpha \mathrm{t}) \mathrm{T}^{2}}}{\exp (-\alpha \mathrm{t})+\exp (-2 \alpha \mathrm{t}) \mathrm{T}} \\
\mathrm{n}=\frac{-(\mathrm{R}+1) \pm 2 \sqrt{\mathrm{R}}}{(\mathrm{R}-1)}
\end{gathered}
$$

In the high photon energy region, the energy dependence of absorption coefficient suggests the occurrence of direct band gap of the crystal obeying the following equation for high photon energies (hv) [8]:

$$
(\alpha h v)^{2}=\mathrm{A}\left(\mathrm{E}_{\mathrm{g}}-\mathrm{hv}\right)
$$

where $E_{g}$ is the optical band gap of the crystal and A is a constant. The variation of $(\alpha h v)^{2}$ with hv [9] in the fundamental absorption region is plotted in the Fig. 5b. The band gap of the crystal was evaluated by extrapolation of the linear part of the graph and found to be $4.0 \mathrm{eV}$. The wide band gap of the urea phosphoric acid crystals confirms the large transmittance in the visible region. Fig. $5 \mathrm{c}$ and $5 \mathrm{~d}$ represent the variation of extinction coefficient and refractive index with respect to photon energy ( 1.2 to $6.5 \mathrm{eV}$ ), respectively. It is observed that the crystal has positive refractive index $(n=1.93$ at $4.14 \mathrm{eV})$ with respect to energy and this indicated the focusing nature of the crystal. Hence, it is cleared that the extinction coefficient depends on the photon energy. The reflectance also increases as the energy increases (Fig. 5e).

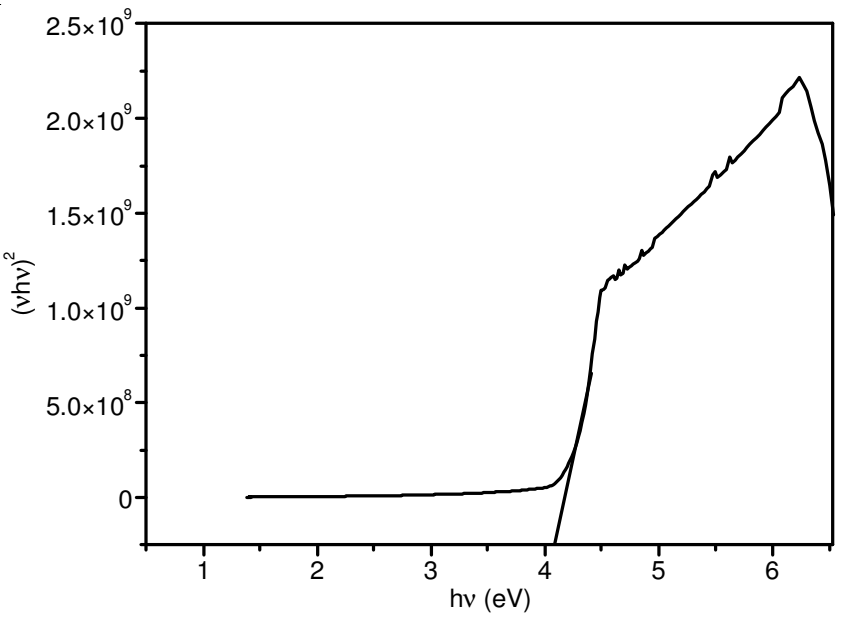

Fig. 5(b). Plot of $(\alpha h v)^{2} v s$. photon energy of urea phosphoric acid crystal

From the optical constants, the electric susceptibility $\chi_{\mathrm{c}}$ can be calculated according to the relation [10]:

$$
\begin{gathered}
\varepsilon_{\mathrm{r}}=\frac{\varepsilon_{0}+4 \pi \chi_{\mathrm{c}}}{4 \pi}=\eta^{2}-\mathrm{K}^{2} \\
\chi_{\mathrm{c}}=\frac{\left(\mathrm{n}^{2}-\mathrm{K}^{2}-\varepsilon_{0}\right)}{4 \pi}
\end{gathered}
$$

where $\varepsilon_{0}$ is the dielectric constant in the absence of any contribution from free carriers. The value of electric susceptibility $\chi_{\mathrm{c}}$ is 0.2874 at $300 \mathrm{~nm}$. 


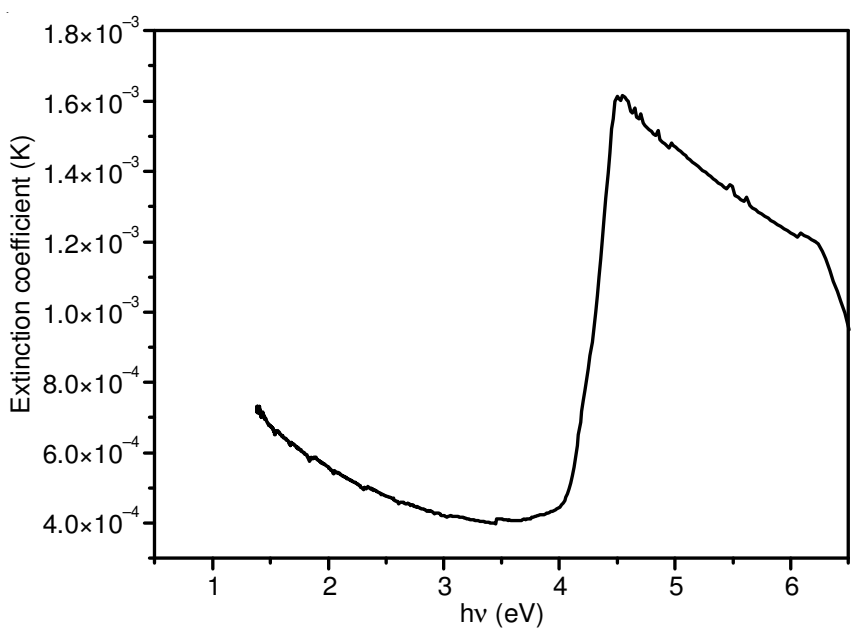

Fig. 5(c). Plot of energy vs. extinction coefficient of urea phosphoric acid crystal

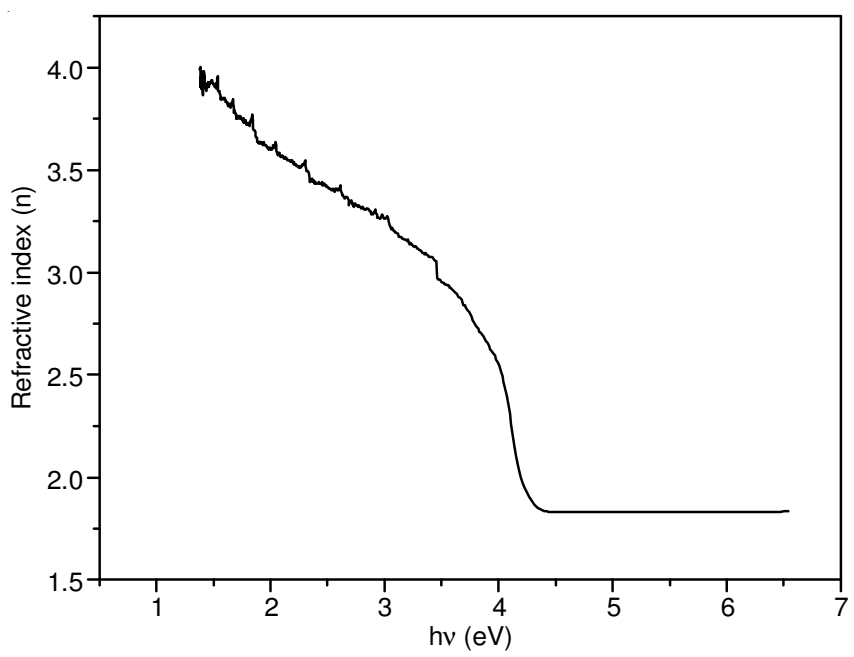

Fig. 5(d). Plot of refractive index vs. photon energy urea phosphoric acid crystal

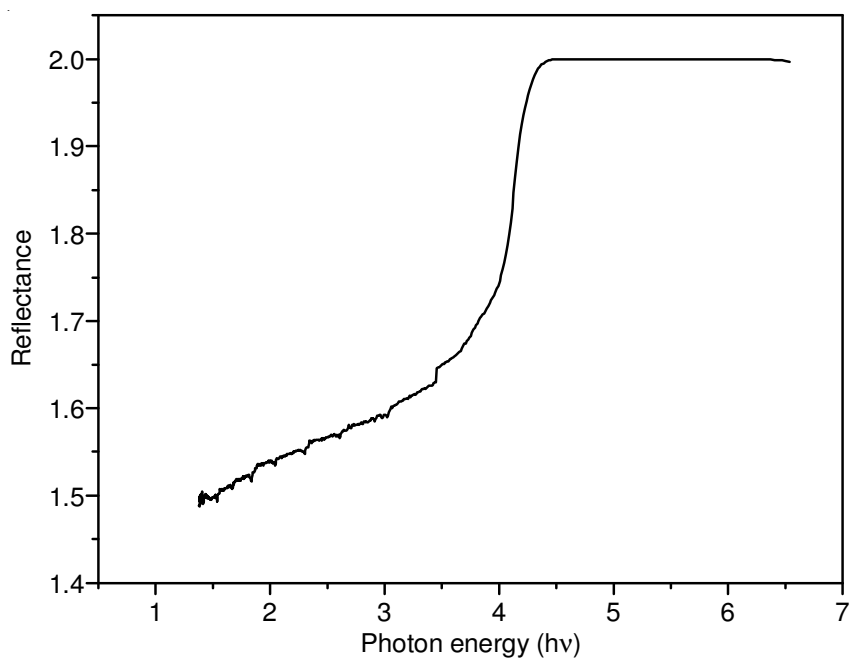

Fig. 5(e). Plot of reflectance vs. photon energy urea phosphoric acid crystal

The real $\left(\varepsilon_{\mathrm{r}}\right)$ and imaginary $\left(\varepsilon_{\mathrm{i}}\right)$ dielectric constants can be calculated from the following relations [11]

$$
\varepsilon_{\mathrm{r}}=\mathrm{n}^{2}-\mathrm{K}^{2} \quad \text { and } \quad \varepsilon_{\mathrm{i}}=2 \mathrm{nK}
$$

The value of real $\varepsilon_{\mathrm{r}}$ and imaginary $\varepsilon_{\mathrm{i}}$ dielectric constants at $\lambda=300 \mathrm{~nm}$ are 3.6099 and $2.071 \times 10^{-3}$ respectively.
Refractive index studies: The refractive index of the urea phosphoric acid crystal was determined by Brewster's angle method. A polished single crystal of urea phosphoric acid with $1 \mathrm{~mm}$ thickness was mounted on a rotating mount at an angle varying from 0 to $90^{\circ}$. The angular reading on the rotary stage was observed, when the crystal was perfectly perpendicular to the intra-cavity beam. The crystal was rotated until the reflection of laser light vanishes and this angle has been noted. Brewster's angle $(\theta p)$ for urea phosphoric acid was measured to be $55^{\circ}$. The refractive index was calculated using the formula $\mathrm{n}=\tan \theta \mathrm{p}$ and found to be 1.48 .

Dielectric studies: The plates were polished and coated with an electronic grade silver paste, which acts as an electrode. Fig. 6 shows the variation of dielectric constant $\left(\varepsilon_{\mathrm{r}}\right)$ with $\log$ frequency. From the plot, it is observed that the dielectric constant is relatively higher at $100 \mathrm{~Hz}$ and further decreases with increase in frequency. This effect can be attributed to the effect of charge distribution by mean carrier hopping on defects. At low frequency the charge on the defects can be rapidly redistributed so that defects closer to the positive side of the applied field become negatively charged, while defects closer to the negative side of the applied field become positively charged. This leads to a screening of the field and overall reduction in the electric field. The high value of dielectric constant at low frequency can be attributed to the lower electrostatic binding strength, arising due to the space charge polarization near the grain boundary interfaces. In accordance with Miller rule, the lower value of dielectric constant at higher frequencies is a suitable parameter for the enhancement of SHG coefficient [12]. The characteristic of low dielectric loss (Fig. 7) with high frequency for the sample suggests that the crystal possess enhanced optical quality with lesser defects and this parameter play a vital role for the fabrication of non-linear optical devices [13].

Microhardness studies: Hardness is the resistance offered by a material to the localized deformations caused by scratching or indentations. The indentation hardness is measured as the ratio of applied load to the surface area of the indentation. Microhardness measurement was made on the well-defined plane of the grown crystal using Vickers' microhardness tester fitted with a diamond pyramidal indenter for loads varying

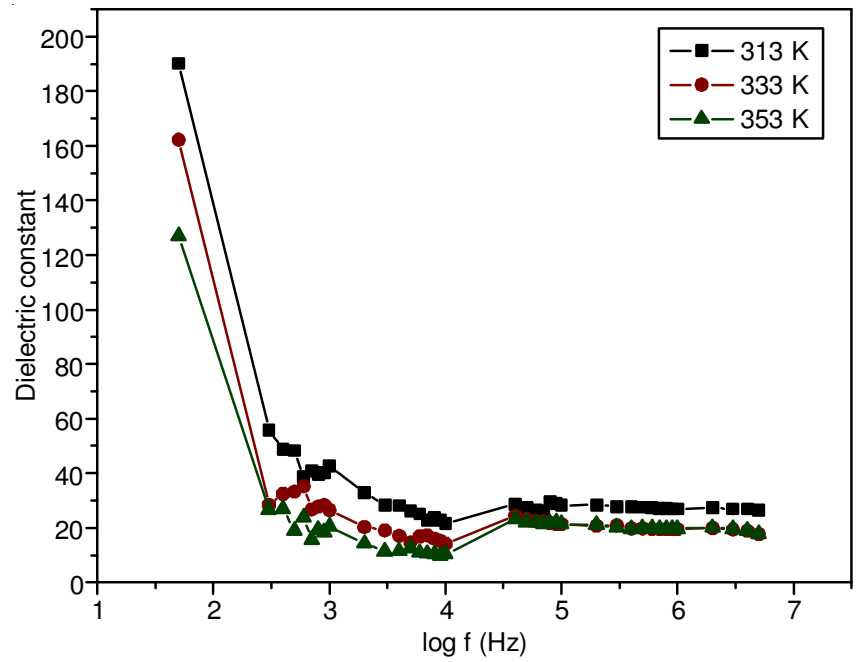

Fig. 6. Plot of frequency $v s$. dielectric constant of urea phosphoric acid crystal 


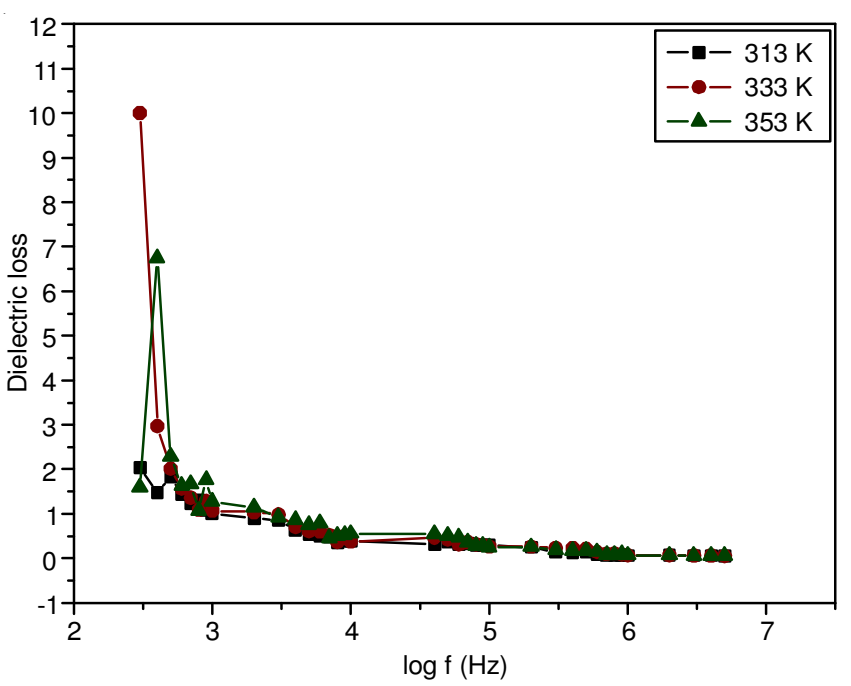

Fig. 7. Plot of frequency vs. dielectric loss of urea phosphoric acid crystal

from 10 to $70 \mathrm{~g}$. The indentation time was maintained constant at $5 \mathrm{~s}$. Vickers' micro hardness number was calculated using the relation:

$$
\mathrm{H}_{\mathrm{v}}=1.8544 \mathrm{P} / \mathrm{d}^{2} \mathrm{~kg} \mathrm{~mm} \mathrm{~m}^{-2}
$$

where $\mathrm{P}$ is the applied load in $\mathrm{kg}$ and $\mathrm{d}$ is the mean diagonal length of the indenter impression in millimeter. It was observed that Vickers' hardness number increases with load. This effect is known as reverse indentation size effect (RISE). However, with increasing load the indentation marks are found to be surrounded by a highly reflecting area, thereby obscuring the actual boundary of the indentation marks. Hence, all the subsequent measurements are performed with the lowest load of 10 $\mathrm{g}$. The measurement performed beyond a load of $50 \mathrm{~g}$ resulted in severe cracks (Fig. 8). This may be due to the release of internal stress generated locally by indentation. From the Meyer's relation, the work hardening coefficient 'n' was found to be 1.4. Hence, urea phosphoric acid crystal belongs to hard material category [14].

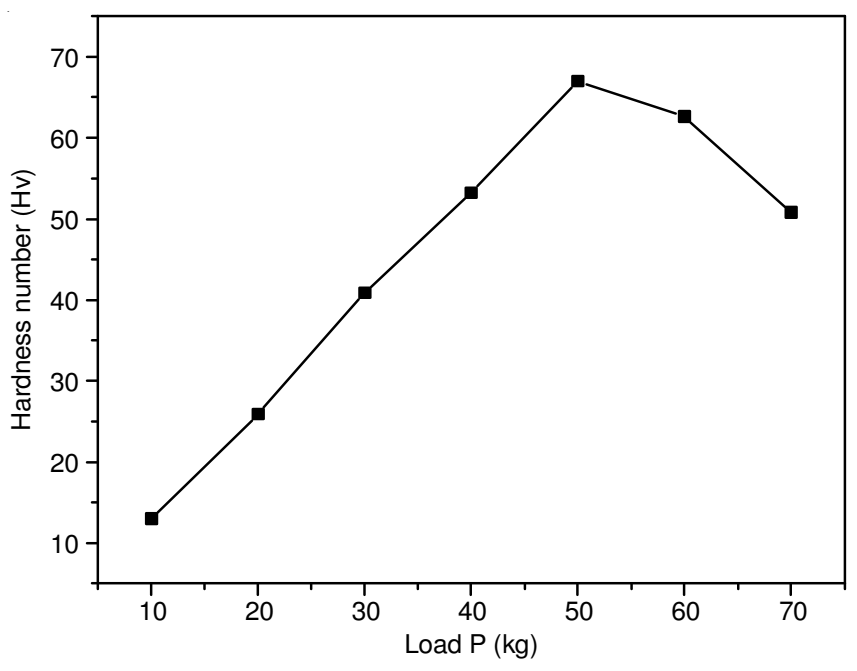

Fig. 8. Load (P) vs. hardness number (Hv) of urea phosphoric acid crystal

Etching studies: For fabrication of optical devices, we need good quality crystals with minimum defects. The quality of the grown crystals is usually assessed by knowing the imperfections, particularly dislocations in crystals. Dislocations influence a number of physical properties like plasticity, mechanical strength, etc. Hence it is necessary to know the density and distribution of dislocations in a crystal. To obtain the structural perfection and growth features of a grown crystal, etching studies can be used. The urea phosphoric acid crystal has been completely immersed for $5 \mathrm{~s}$ in the water etchant and the sample was wiped out with dry filter paper. Using a Magnus MXL Microscope the features of the crystal has been analyzed which is shown in Fig. 9. From Fig. 9, number of etch pits have been identified with identical shape. The patterns reveal square etch pits and these faces possess minimum fourfold rotational symmetry [15].

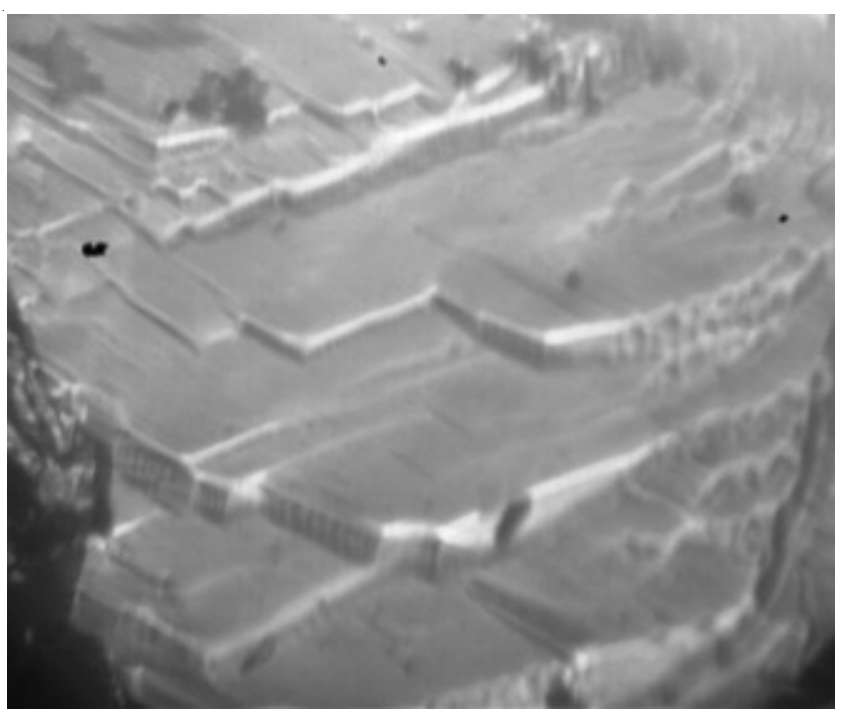

Fig. 9. Etch pattern of urea phosphoric acid crystal with water as an etchant

Laser damage threshold studies: The operation of nonlinear optical (NLO) devices clearly involves the exposure of these materials to high power laser light. Hence, the utility of NLO crystal depends not only on the linear and NLO properties but also largely on its ability to withstand high-power lasers [16]. Laser damage studies on NLO crystals are extremely important as the surface damage of the crystal by high-power lasers limits its performance in non-linear optical applications [17]. In the present investigation, laser damage threshold studies have been carried out for the solution grown urea phosphoric acid crystals using a Q-switched Nd:YAG laser of pulse width $8 \mathrm{~ns}$ at a wavelength of $1064 \mathrm{~nm}$ and a $10 \mathrm{~Hz}$ repetition rate operating in TEM00 mode, is used as the source. The output intensity of the laser was controlled with a variable attenuator and delivered to the test sample located at the near focus of converging lens. The lens with a focal length of 30 $\mathrm{cm}$ was used, which helped in setting the spot size to the desired value. During laser radiation, the power meter records the energy density of the input laser beam at which the crystal gets damaged. In the present study, the laser damage threshold value of urea phosphoric acid crystal was found to be $6.3 \mathrm{GW} /$ $\mathrm{cm}^{2}$. The laser damage threshold values of some NLO organic and inorganic single crystals are given in Table- 1 for the sake of comparison. 
TABLE-1

COMPARISON OF LASER DAMAGE THRESHOLD VALUE

OF UREA PHOSPHORIC ACID WITH SOME INORGANIC, ORGANIC AND SEMIORGANIC CRYSTALS

\begin{tabular}{lcc}
\hline \multicolumn{1}{c}{ Crystal } & $\begin{array}{c}\text { Laster damage } \\
\text { threshold }\left(\mathrm{GW} / \mathrm{cm}^{2}\right)\end{array}$ & Ref. \\
\hline Urea & 1.5 & {$[18]$} \\
Benzimidazole & 2.9 & {$[18]$} \\
$\beta$-Barium borate & 5.0 & {$[18]$} \\
L-Prolinium tartrate & 5.9 & {$[19]$} \\
L-Tartaric acid & 5.4 & {$[6]$} \\
Urea phosphoric acid & 6.3 & Present work \\
\hline
\end{tabular}

Photoconductivity studies: Photoconductivity measurements are carried out on a polished ( $\left.\begin{array}{lll}0 & 1 & 0\end{array}\right)$-plane of the urea phosphoric acid crystal by fixing it onto a microscope slide. The sample is connected in series with a diffractive curve power supply and KEITHLEY 485 picoammeter. The sample is covered with a black cloth and the voltage applied is increased from 0 to $500 \mathrm{~V}$ in steps of $50 \mathrm{~V}$, the dark current is recorded. The sample is illuminated by the radiation from $100 \mathrm{~W}$ halogen lamp containing iodine vapour and tungsten filament. The photocurrent is recorded for the same values of the applied voltage. Fig. 10 shows the variation of both dark current and photo current with applied field. It is seen from the plots that both the dark current and photocurrent of the sample increase linearly with the applied field. The photocurrent is seen to be less than the dark current for the same applied field, which is termed as negative photoconductivity. The negative photoconductivity exhibited by the sample may be due to the reduction in the number of charge carriers in the presence of radiation $[20,21]$. The decrease in mobile charge carriers during negative photoconductivity can be explained by the Stockmann model also, which is already reported elsewhere [22].

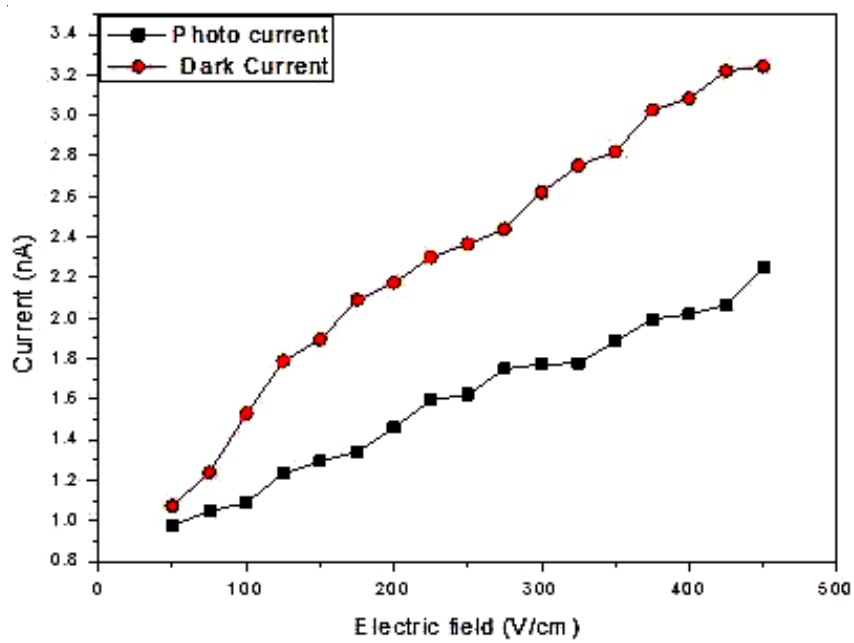

Fig. 10. Plot of electric field vs. current of urea phosphoric acid crystal

SHG measurments: The Kurtz and Perry technique was used to find the non-linear property of the crystal. Microcrystalline KDP material was used as a reference material with urea phosphoric acid for SHG measurements. A high intensity $\mathrm{Nd}$ :YAG laser beam with input pulse of $6.2 \mathrm{~mJ}$ as a optical source was allowed onto the powder samples. The second harmonic signal $(532 \mathrm{~nm}) 56 \mathrm{mV}$ and $78.4 \mathrm{mV}$ were obtained through KDP and urea phosphoric acid samples. Thus the SHG efficiency of the urea phosphoric acid crystal is 1.4 times higher than KDP crystal. This result confirms the non centrosymmetric structure and NLO behaviour of the as grown urea phosphoric acid crystal.

\section{Conclusion}

An organic urea phosphoric acid single crystal has been grown by slow evaporation solution growth technique. The cell parameters of urea phosphoric acid single crystal and its perfection were confirmed by single and high resolution X-ray diffraction analyses. The presence of functional groups was identified by FT-IR spectral analysis. The UV-visible spectral studies showed that the urea phosphoric acid single crystal has wide transmission window in the entire visible region (200$900 \mathrm{~nm}$ ). The dielectric property of urea phosphoric acid crystal revealed the normal dielectric behaviour. From the mechanical measurements, it was observed that the urea phosphoric acid crystal belongs to hard material category. The laser damage threshold value of urea phosphoric acid crystal was found to be $6.3 \mathrm{GW} / \mathrm{cm}^{2}$ by Nd:YAG laser. Photoconductivity studies show the negative photoconductivity. The second order nonlinear coefficient of urea phosphoric acid crystal was 1.4 times higher than KDP.

\section{ACKNOWLEDGEMENTS}

One of the authors (D. Jayalakshmi) is grateful to University Grants Commission, New Delhi, India for financial support under minor research project scheme.

\section{REFERENCES}

1. J. Badan, R. Hierle, A. Perigand and J. Zyss, in ed: D.J. Williams, Nonlinear Optical Properties of Organic Molecules and Polymeric Materials, American Chemical Society, Symposium Series, Washington, DC, D.5, p. 233 (1993).

2. D.S. Chemla and J. Zyss, Nonlinear Optical Properties of Organic Molecules and Crystals, vol. 1, Academic Press, London (1987).

3. R.W. Munn and C.N. Ironside, Principles and Applications of Nonlinear Optical Materials, Chapman and Hall, London (1993).

4. S.R. Marder, J.W. Perry and W.P. Schaefer, Science, 245, 626 (1989); https://doi.org/10.1126/science.245.4918.626.

5. S.R. Marder, J.W. Perry and W.P. Schaefer, J. Mater. Chem., 2, 985 (1992); https://doi.org/10.1039/JM9920200985.

6. S.A. Martin Britto Dhas, M. Suresh, G. Bhagavannarayana and S. Natarajan, J. Cryst. Growth, 309, 48 (2007); https://doi.org/10.1016/j.jcrysgro.2007.09.008.

7. G. Smith and U.D. Wermuth, Acta Crystallogr. C, 66, o5 (2010); https://doi.org/10.1107/S0108270109049154.

8. A. Ashour, N. El-Kadry and S.A. Mahmoud, Thin Solid Films, 269, 117 (1995); https://doi.org/10.1016/0040-6090(95)06868-6.

9. J. Tauc, R. Grigorovici and A. Vancu, Phys. Status Solidi, B Basic Res., 15, 627 (1966); https://doi.org/10.1002/pssb.19660150224.

10. V. Gupta and A. Mansingh, J. Appl. Phys., 80, 1063 (1996); https://doi.org/10.1063/1.362842.

11. M.A. Gaffar, A.A. El-Fadl and S.B. Anooz, Physica B, 327, 43 (2003); https://doi.org/10.1016/S0921-4526(02)01700-3.

12. C. Miller, Appl. Phys. Lett., 5, 17 (1964); https://doi.org/10.1063/1.1754022.

13. C. Balarew and R. Duhlev, J. Solid State Chem., 55, 1 (1984); https://doi.org/10.1016/0022-4596(84)90240-8.

14. E.M. Onitsch, Mikroskopie, 95, 12 (1950).

15. P.V. Dhanaraj and N.P. Rajesh, Physica B, 406, 12 (2011); https://doi.org/10.1016/j.physb.2010.09.041. 
16. G.C. Bhar, A.K. Chaudhary and P. Kumbhakar, Appl. Surf. Sci., 161, 155 (2000); https://doi.org/10.1016/S0169-4332(00)00276-2.

17. N.L. Boling, M.D. Crisp and G. Dube, Appl. Opt., 12, 650 (1973); https://doi.org/10.1364/AO.12.000650.

18. N. Vijayan, G. Bhagavannarayana, T. Kanagasekaran, R.R. Babu, R. Gopalakrishnan and P. Ramasamy, Cryst. Res. Technol., 41, 784 (2006); https://doi.org/10.1002/crat.200510669.
19. S.A.M. Britto Dhas and S. Natarajan, Cryst. Res. Technol., 42, 471 (2007); https://doi.org/10.1002/crat.200610850.

20. R.H. Bube, Photoconductivity of Solids, Wiley, New York (1981).

21. S. Pandi and D. Jayaraman, Mater. Chem. Phys., 71, 314 (2001); https://doi.org/10.1016/S0254-0584(01)00285-1.

22. V.N. Joshi, Photoconductivity, Marcel Dekker, New York (1990). 